\title{
Quake catalogs from an optical monitoring of an interfacial crack propagation
}

\author{
M. Grob ${ }^{1,2}$, J. Schmittbuhl ${ }^{1,2}$, R. Toussaint ${ }^{1,2}$, L.Rivera $^{1,2}$ \\ S. Santucci ${ }^{3}$, K. J. Måløy ${ }^{3}$
}

March 31, 2008

\begin{abstract}
Using an experimental setup which allows to follow optically the slow propagation of an interfacial crack front in a heterogeneous medium, we show that the fracture front dynamics is governed by local and irregular avalanches with large velocity fluctuations. Events defined as high velocity bursts, are ranked in catalogs with analogous characteristics to seismicity catalogs: time of occurence, epicenter location and energy parameter (moment). Despite differences in the fracturing mode (opening for the experiments and shear rupture for earthquakes), in the acquisition mode and in the range of timescales, the distributions of moment and epicenter jumps in the experimental catalogs obey the same scaling laws with similar exponents as the corresponding distributions for earthquakes. The record breaking event analysis shows also very strong similarities between experimental and real seismicity catalogs. The results suggest that the dynamics of crack propagation is controlled by the elastic interactions between microstructures within the material.
\end{abstract}

\footnotetext{
${ }^{1}$ EOST, Université Louis Pasteur, 5 rue René Descartes, F-67084 Strasbourg Cedex, France.

E-mail: melanie.grob@eost.u-strasbg.fr

${ }^{2}$ Institut de Physique du Globe de Strasbourg, UMR CNRS 7516, 5 rue René Descartes, F-67084 Strasbourg Cedex, France

${ }^{3}$ Physics Institut, University of Oslo, PO Box 1048 Blindern, N-0316 Oslo, Norway
} 


\section{Introduction}

In observational seismology, attempts to characterize earthquake ruptures have motivated numerous studies from the very early stages (Reid (1910)). The description of small earthquakes is often reduced to a source point description given by a set of simple parameters: time of occurrence, source location, seismic moment or magnitude, possibly focal mechanism (Clinton et al. (2006)). Despite this crude information, organized in well-know seismic catalogs, these simple parameters have provided extremely useful earthquake features like the GutenbergRichter magnitude-frequency relationship (Gutenberg and Richter (1944)). A complementary approach is to analyse the properties of interactions between earthquakes (Bak et al. (2002), Kagan (2002), Ziv et al. (2003), Corral (2004), Davidsen and Paczuski (2005)). It has been shown for instance that the spatiotemporal evolution of earthquakes in a specific region exhibits a fractal pattern resulting of a dynamical process and expressing a hierarchical organization of events in space and time (Marsan et al. (2000), Davidsen et al. (2006)).

Seismic inversions of slip history during large earthquakes have been an important step in the description of the rupture process (e.g. Wald et al. (1991); Bouchon et al. (2000)). The complete fault region associated to the earthquake is then considered. Slip history inversions provide important features of the rupture propagation: fault geometry, rupture length, rupture speed, time rise, rupture width, etc. However they rely on strong assumptions like simple fault models for kinematic inversions (Kikuchi and Kanamori (1991)) or simple friction processes for dynamic inversion (Ide and Takeo (1997), Fukuyama et al. (2003)) and subsequently provide low resolution or speculative images of the rupture complexity. Statistics from these inversions however allow more precise statistical relationships like the ones linking the seismic moment $M_{0}$ to the three geometric quantities characterising the rupture: its length $L$, its width $W$ and the average slip on the fault $u$ (Kanamori and Anderson (1975), Wells and Coppersmith (1994), Mai and Beroza (2000)). In general, all these relationships can be approximated by a power law and exponents are found to be mostly universal (Scholz (2002), Kagan (1999)). New tools to account for the full complexity of the earthquake rupture are required and under development (Mai and Beroza (2002), Aochi and Fukuyama (2002), Tinti et al. (2005), Rubin and Ampuero (2007)).

Fracture complexity has also been addressed in the mechanical community in particular since the pioneer work of Mandelbrot et al. (1984). In particular, scaling properties of fractures 
in heterogeneous materials were discovered (Bouchaud (1997)). For instance, the roughness of fracture surfaces in heterogeneous media exhibits a self-affine morphology. The associated roughness exponent is found to be very robust for different materials such as steel or aluminium alloy (Bouchaud et al. (1993)) or fault rocks (Schmittbuhl et al. (1993), Schmittbuhl et al. (1995)). Experiments were also carried out to investigate the interactions between the crack front and the material heterogeneities (Daguier et al. (1995)). In a simplified two dimensional configuration, studies of the crack front that is constrained geometrically in a plane), both theoretically (Schmittbuhl et al. (2003a), Schmittbuhl et al. (2003b)) and experimentally (Schmittbuhl and Måløy (1997), Delaplace et al. (1999)) were performed. The front line morphology is shown to exhibit scaling invariances (Schmittbuhl and Måløy (1997), Delaplace et al. (1999), Santucci et al. (2007)). More recent studies were performed to describe the crack front dynamics (Schmittbuhl et al. (2001), Santucci et al. (2006), Måløy et al. (2006)). These works show that the crack propagation is controlled by the pinning and depinning of the front owing to local asperities. The fracture front dynamics is then governed by local and irregular avalanches of varying sizes and velocities.

In this article, we propose a link between the fracture dynamics analysed at the laboratory scale and the earthquake dynamics extracted from a subset of the Southern California catalog (SHLK catalog). The core of the study is the construction of laboratory scale catalogs of events or quakes from an optical monitoring of an interfacial crack front propagation in a heterogeneous medium. Using a transparent material (Plexiglas), the experimental setup allows to follow visually at high spatial resolution and continuously in time, the rupture process. The first part of the paper describes the sample preparation and how the experimental setup works. The next section explains the image processing of the raw crack front pictures in order to obtain the parameters needed to build the quake catalogs made of several thousands of events. Since we aim at comparing our experimental results to natural seismicity data, we show in a following section, the results of statistical tools, typically applied to natural seismicity data, when applied to the experimental data. Finally a discussion of the reliability of analogies or differences between experimental and natural data is proposed. 


\section{An interfacial rupture experiment}

\subsection{Sample preparation}

The samples are built from two types of transparent polymethylmethacrylate (PMMA) plates: the main plate is $16 \mathrm{~cm}$ long, $14 \mathrm{~cm}$ large and $1 \mathrm{~cm}$ thick; the other ones are a set of $17 \mathrm{~cm}$ long, $0.4 \mathrm{~cm}$ thin and $1 \mathrm{~cm}$ wide bands. One surface of each plate is first sand-blasted with glass beads of diameter $\varnothing=200 \mu \mathrm{m}$ or $500 \mu \mathrm{m}$. The thick plate is then assembled to a set of four thin plates (see Fig. 1(a)), sand-blasted surfaces facing each other, and placed under a normal homogeneous load, so that the remaining air is expelled from the contact area when the PMMA sandwich is loaded. The loading frame for the sample preparation is made of two parallel aluminium plates between which the Plexiglas plates sandwich is placed. Twelve screws are used to exert a normal load on the sample (see Fig. 1(b)). The torque applied when tightening the screws is equal to 10 N.m.

To anneal the plate assembly, the whole block (sample + press) is put in an oven at $205^{\circ} \mathrm{C}$ during 30 minutes, which allows to stick the Plexiglas plates together. The thermal annealing process produces a cohesive interface along the former boundary between the two plates. This interface, weaker than the bulk PMMA, constrains the block to break along an interfacial fracture during mode I crack. Figure 1 summarizes this sample preparation procedure.

An important feature of the interface preparation is the sand-blasting procedure that induces a random topography on the PMMA surface to be annealed and accordingly controls the local toughness during annealing. Another consequence of the sand-blasting technique is that the transparency of the Plexiglas plates is lost: light scatters because of the microstructures introduced by the sand-blasting. But after annealing, the newly formed block recovers its transparency since contrast of the refraction index along the interface disappears.

\section{Figure 1}

\subsection{Setup}

The thick PMMA plate is clamped to a stiff aluminium frame. The thin plates are pulled apart thanks to a rod that presses on the excess length of the thin Plexiglas bands. A diagram of the setup is displayed in figure 2. The loading translation stage is controlled by a stepping motor that applies a continuous descent of the rod. The normal displacement imposed on the 
Plexiglas band induces a stable crack propagation in mode I within the artificially introduced weak plane of the sample that is followed optically with an optical microscope. Using a high speed camera mounted on top of the microscope, up to 12288 consecutive images of the crack advance can be recorded with a spatial resolution of 1024 X 512 pixels $(1$ pixel $\approx 10 \times 10$ $\mu m^{2}$ ) at an acquisition rate of 1000 frames per second (fps) which corresponds to an acquisition duration of 12 seconds. To provide sufficient light intensity at this small closure time (1 ms), the sample is lighted from below using optical fibers linked to an intense light source. These optical fibers contain lens elements on their end that allow to focus the light source onto the crack front. The width of the image acquired by the fast camera is adjusted to the width of the PMMA band. After opening of the sample, light re-scatters in the fractured part of the sample because of the microstructures previously introduced on the PMMA plates. The open part of the sample looks opaque whereas the sintered region is still transparent. The transition between these two areas corresponds to the crack front (see Fig. 3(a)).

\section{Figure 2}

\section{$3 \quad$ Image processing}

\subsection{Front extraction}

Images of the interfacial fracture are divided in two parts: a bright zone which is the cracked and open area (microstructures along the interface scatter the light of the newly open surface) and a dark region which is still soldered and transparent. The image processing aims at extracting the shape of the transition zone between the bright and dark areas of the picture which corresponds to the crack front. Some white spots ahead of the fracture front appear on the image (see the dark part of the photo in figure $3(\mathrm{a})$ ). This is due to flaws created by the sand-blasting but located shallow inside the Plexiglas plates which scatter the light. As an attempt to remove these flaws in the fracture front images, a subtraction of a background picture taken before any opening is performed systematically. An example of the resulting image is displayed in figure 3(b). Some flaws are still visible but the contrast has been markedly enhanced. The gray level histogram of the image is clearly bimodal (Fig. 3(c)): the lobes represent the two different regions of the image. The picture is then clipped at the threshold level that separates these two lobes (Fig. 3(d)). The norm of the gradient of the segmented image is computed to search for 
the boundaries (Fig. 3(e)). Isolated clusters of pixels that might appear are removed by keeping only the largest cluster that percolates from the left to the right edge of the picture (Fig. 3(f)) to obtain the front path. More details about the last four steps of the image processing can be found in Delaplace et al. (1999). Figure 3(g) shows the extracted front superimposed on the raw image. A zoom on the preceding picture is done to show the resolution of the extracted front (cf figure $3(\mathrm{~h})$ ). Its irregular shape is explained by the fact that the front is pinned by local zones of high toughness during the propagation and becomes rough.

\section{Figure 3}

\subsection{Quake definition}

In order to analyse the burst dynamics of the interfacial crack propagation and define rupture quakes, the fracture front lines extracted from image processing are added to form the waiting time matrix W(x,y) (Måløy et al. (2006), Måløy et al. (2005)). This matrix has the same size as the raw images and initial values equal to zero for all its elements. The value 1 is added to the matrix element $w$ corresponding to pixels where a front line position $(\mathrm{x}, \mathrm{y})$ is detected. If several successive fronts are observed at the same particular position, the matrix element $w$ at this position increases by one for each encountered front. This procedure was done for all frames of a given experiment to obtain the final waiting time matrix $\mathbf{W}(\mathbf{x}, \mathbf{y})$ (Fig. $4(\mathrm{a}))$. The waiting times estimate the amount of time during which the front was stuck at a precise position. The local speed of the crack front at the time when it passes a particular position $(\mathbf{x}, \mathbf{y})$ can be deduced from $\mathbf{W}(\mathbf{x}, \mathbf{y})$ by the inverse value of the corresponding matrix element $w$ multiplied by the ratio of the pixel linear size $a$ (typically $10 \mu \mathrm{m}$ ) over the typical time $\delta t$ between two pictures (0.001 s). The velocity matrix $\mathbf{V}(\mathbf{x}, \mathbf{y})$ is therefore defined as

$V(x, y)=\frac{1}{W(x, y)} \frac{a}{\delta t}$, thus associating a local crack front speed to each pixel of the crack front in each image. It is important to mention that the image recording is performed so fast that there is basically no hole in the waiting time matrix, i.e. no element equal to zero (except below the first front, above the last one, and a few artefacts due to initial flaws in the sample).

In order to define events (or quakes), the velocity matrix $\mathbf{V}(\mathbf{x}, \mathbf{y})$ is clipped by setting its elements $v$ equal to 1 if $v>C\langle v\rangle$ and 0 elsewhere, where $C$ is a constant of the order of a few unities (Måløy et al. (2006), Måløy et al. (2005)). Figure 4(b) is an example of the thresholded matrix with $C=10$. The white clusters correspond to regions where the crack front speed 
is 10 times larger than the average front velocity $\langle v\rangle$. The clusters connected to the first and last fronts, belonging to the upper and lower white zones are excluded from the analysis. Each cluster is considered as an event or quake.

\section{Figure 4}

\subsection{Quake catalogs}

This data processing is applied to two experiments (named A and B) with different parameters. Table 1 summarizes the parameters for both experiments. Three different $\mathrm{C}$ values are used to threshold the velocity matrix corresponding to each experiment. We thus have six data sets of events. The aim now is to build catalogs from these data sets that could be compared to real seismicity data catalogs. The mostly used quantities are: the time of occurrence, the location of hypocenter and an energy parameter of the event (seismic moment or magnitude).

For each event of each data set, the time of occurrence is found by searching for the first pixel in time that is attached to the considered cluster. The time of occurrence $t$ in $\mathrm{ms}$ is calculated considering the beginning of the experiment as the original time.

The epicenter of an event is given by the position $(x, y)$ of this first pixel. As the crack front propagation is constrained in a plane, the $z$ coordinate is considered constant and equal to 0 in a first approximation. As the $y$ axis is the direction of propagation of the crack front, a strong link exists between the time and the $y$ coordinate of the epicenter. Hence the spatial position of the event is mainly given by the $x$ coordinate.

The moment $M$ of an event is given by the area of the event (which is the total number of pixels belonging to the cluster) multiplied by a characteristic opening which is supposed to be constant. The parameter $M$ is then directly proportional to the area of the event. The three parameters $t$, the couple $(x, y)$ and $M$, build up a quake catalog for our experiments. In total, six quake catalogs named A1, A2, A3, B1, B2 and B3 were created from the velocity matrix of two different experiments A and B, each thresholded with three various level values (table 1).

Figure 5 shows the locations of the epicenters of events superimposed on the thresholded velocity matrix for experiment A2. The diameter of the circles representing the quakes is proportional to the logarithm of the area of the clusters. Only events with $\log _{10}(M)>1.5$ are displayed for a better visibility. The good agreement between the circles and the corresponding cluster contributes to validate the method used to build the catalogs. For a better readability, 
the logarithm of the moment $M$ is denoted $m$ in the following.

\section{Table 1}

\section{Figure 5}

\subsection{Quake maps}

As first qualitative analyses, some maps are drawn from the catalogs. Figure 6 shows the locations of epicenters for the six experimental catalogs. The diameter of the circles is proportional to $m$. Differences can be seen between the three experimental catalogs generated from the same experiment concerning the size of the events and their localisation. They are due to the removal of pixels when increasing the $\mathrm{C}$ level, thus dividing some large clusters in smaller ones. The density of events is higher for experiment B than for experiment A. This may be related to the velocity of propagation of the crack front which is larger for experiment B. An effect of clustering can be observed on the maps: some gaps appear in the distribution of the events.

\section{Figure 6}

Another way of showing a possible effect of clustering is to display events on a time versus $x$ coordinate map. Figure 7 represents this spatio-temporal distribution of events (diameters of circles are proportional to $m$ ). The clustering effect is clearly visible on both maps: several events follow each other in time in a small zone along the $\mathrm{x}$-axis. This means that the advancing of the crack front is very irregular and controlled by the ability of the front to go through local region of high toughness. Indeed the front is stopped in some places because of local high toughness bounds on the interface. When it manages to go through, the front can advance over some distance with a high velocity, which helps breaking other bounds, before being stuck again.

\section{Figure 7}




\section{Comparison between experimental results and real seis- micity data}

\subsection{The SHLK catalog}

To compare our experimental catalogs and real seismicity data, a subset of the Southern California catalog is examined: the SHLK catalog (data available at http://www.data.scec.org/ftp/catalogs/SHLK This catalog is supposed to be homogeneous from January 1984 to December 2002 and complete for earthquakes with magnitude larger than 2.5 in the area $\left(120.5^{\circ} \mathrm{W}, 115.0^{\circ} \mathrm{W}\right) \times\left(32.5^{\circ} \mathrm{N}, 36.0^{\circ} \mathrm{N}\right)$, giving 22217 events to be considered. A detailed description of the catalog can be found in Shearer et al. (2005).

The exact date and time of occurrence of an earthquake is given with a precision up to a thousandth of a second. The time of occurrence of events in the experimental catalogs is also precise up to $1 \mathrm{~ms}$, but the real seismicity data catalog covers almost twenty years whereas the experiments only last a few seconds. The location of hypocenters is specified by three coordinates: latitude, longitude and depth. The configuration is clearly three-dimensional on the contrary to the experiment where the coordinates $(x, y, z)$ simplify to the couple $(x, y)$ because $z$ is constant. But a parallel can be made if all the earthquakes in the catalog are assumed to occur on the same fault plane. Concerning the energy parameter, only the magnitude was available in the chosen catalog. The seismic moment (units in N.m) was calculated with the following relationship between seismic moment and magnitude:

$$
\log _{10}\left(M_{0}\right)=\frac{3}{2} M_{w}+9.1
$$

where $M_{w}$ is the so called moment magnitude (Kanamori (1977)) given in the catalog. The seismic moment is a quantity that combines the cracked area $S$ with the amount of fault offset $D$ and the shear modulus $\mu: M_{0}=\mu S D$. The analogy between the moment defined for the events in the experiments and the seismic moment is discussed in the last section of this paper.

\subsection{Moment distribution}

Figure 8(a) shows the non cumulative quake size distributions $P(M)$ for the six experimental catalogs. A log binning was used to build the distributions which were also normalized by the total number of events for a better comparison of all the data. A robust result is found for both 
experiments and for the different values of $\mathrm{C}$ as all the data collapse. The power law behaviour of the distribution $P(M) \propto M^{-\gamma}$ proves that the burst dynamics occurs at all length scales. A fit of the data to a power law function gives a slope equal to 1.7. A large scale cut-off appears for all the curves but at different $m$ values, due to the lack of statistics. The exponent $\gamma=1.7$ is comparable to exponents already found in earlier works for experiments based on the same mechanical setup over a wider range of experimental conditions (Måløy et al. (2006)). The authors in (Måløy et al. (2006)) used samples sand-blasted with smaller sized beads than the ones used for the experiments described in this paper and the average front line speeds they found were also much smaller $\left(0.3 \mu \mathrm{m} . \mathrm{s}^{-1} \leq\langle v\rangle \leq 40 \mu \mathrm{m} . \mathrm{s}^{-1}\right)$. But the fact that the value of the exponent determined for our new data is similar to the results of former experiments from Måløy et al. (Måløy et al. (2006)) contributes to validate the technique.

\section{Figure 8}

As a first comparison between our experimental catalogs and real seismicity data, figure 8(b) displays the non cumulative seismic moment distribution $P\left(M_{0}\right)$ of earthquakes taken from the SHLK catalog. The power law behaviour of $P\left(M_{0}\right)$ can be related to one well known scaling law in seismology, the Gutenberg-Richter relationship (Gutenberg and Richter (1944)) between seismic moment $M_{0}$ and frequency of earthquakes:

$$
P\left(M_{0}\right) \propto M_{0}^{-1-\beta}
$$

where $P\left(M_{0}\right)$ is the frequency-moment distribution. The value $\beta$ was found to equal $\frac{2}{3}$ and proven to be very robust over different regions (Gutenberg and Richter (1944), Scholz (2002)). Hence the exponent $\gamma=1.7$ found for the experimental catalogs is similar to the exponent of the Gutenberg-Richter relationship (equation 2) for earthquakes of the SHLK catalog.

\subsection{Epicenter jump distribution}

After having analysed the size distribution of events, we focus on the spatial distances or "jumps", $\Delta r_{i}=\sqrt{\left(x_{i+1}-x_{i}\right)^{2}+\left(y_{i+1}-y_{i}\right)^{2}}$ between successive events with epicenter coordinates $\left(x_{i}, y_{i}\right)$ and $\left(x_{i+1}, y_{i+1}\right)$. Following the work of Davidsen et al. (Davidsen and Paczuski (2005)), the probability density function of the jumps $P_{L}(\Delta r)$ is measured for the six experimental catalogs. The result can be seen in figure 9. The straight line with a slope equal to 0.6 is displayed as a guide to the eye. A cut-off is reached around $r \approx L / 2$ because of the 
finite spatial size of the considered areas. Davidsen et al. (Davidsen and Paczuski (2005)) applied this method to characterize spatial clustering of earthquakes to the SHLK catalog. The distribution of jumps for the Californian catalog is superimposed to the experimental ones in the graph 9 (black triangle curve). It decays like the other curves with a trend also following a power law with exponent $\gamma=0.6$. Despite some differences in the coordinates of hypocenters (the $y$ coordinate in the experimental catalogs is closely related to the time and hypocenters of earthquakes are given for a 3-D configuration), a good correlation between the distributions of jumps for experimental events and real earthquakes can be observed.

\section{Figure 9}

\subsection{Record breaking event analysis}

Another comparison between experimental and real data is done with a method developed by Davidsen et al. (Davidsen et al. (2006)). They introduce the notion of record breaking event. An event $B$ is considered as a record with respect to an earthquake $A$ if no event happens in the spatial disc with radius $\overline{A B}$ centered on A during the time interval $\left[t_{A}, t_{B}\right]$ with $t_{A}<t_{B}$. Each record $\mathrm{B}$ is characterized by the distance $l=A B$ and the time interval (or waiting time) $T=t_{B}-t_{A}$ between the two earthquakes. Each event in the catalog has its own sequence of records (or recurrences). Davidsen et al. tested this method on the SHLK catalog. They found that the probability density functions of distances $l$ and of recurrence times $T$ both follow power laws over a few decades.

An analogy can be made here between our experiment and real seismology. Our mode I setup has to be compared to a mode II fault in friction: the y-axis in the experiments is related to the time for the slipping fault (the y coordinate value would represent a slip), while the $\mathrm{x}$-axis has to be linked with space. The catalogs built from the experiments were processed in order to obtain a sequence of records for each cluster event but considering only the $\mathrm{x}$ coordinate as a position of the epicenters. Figure 10(a) shows the probability density function $P_{x}\left(l_{x}\right)$ of distances $l_{x}$ of recurrent events for the different experiments. All distributions collapse and follow a power law decay over several decades with an exponent $\delta \approx 1.1$. This value of the slope is close to the value found by Davidsen et al. (Davidsen et al. (2006)) for the Southern California seismicity catalog $(\approx 1.05)$. The probability density functions are independent of the experiments and the threshold level $\mathrm{C}$ proving the robustness of the process. The cut-off 
appearing between 4 and $5 \mathrm{~mm}$ is induced by the finite size of the system. The limit of linearity for the left side of the curves is due to location errors of the epicenters (distances below $40 \mu \mathrm{m}$, which corresponds to 4 pixels, are unreliable). The record breaking event analysis was also applied to three experimental catalogs (A2, B1 and B3) considering only events with a moment parameter larger or equal to a certain value of $m$. Figure 10(b) show the distributions $P_{x, m}\left(l_{x}\right)$ resulting from this analysis. The black straight line is a reminder of the power law with an exponent $\delta=1.1$. The linear part of the curves for intermediate distances follows this power law behaviour. The cut-off at $4-5 \mathrm{~mm}$ is still observable but the peaks of the curves on the left side now vary a little with the moment (the larger the moment threshold, the larger the minimum distance limit).

\section{Figure 10}

The distributions $P_{x}\left(T_{x}\right)$ for the time intervals $T_{x}$ were also calculated for the record breaking events obtained considering only the x position of the epicenters. They are shown in figure 11(a). They obey a power law with an exponent $\alpha_{x}=0.95$ when fitting the data for the intermediate values of recurrent times. Davidsen et al. (Davidsen et al. (2006)) found an exponent $\alpha \approx 0.9$, which is very close to our result. The variations from the general trend appearing for small $T$ (between 3 and $4 \mathrm{~ms}$ ) are due to inaccuracy concerning the time of occurrence of the events. The cut-off for large $T$ indicates an upper limit of the waiting times. Figure 11(b) displays the distributions $P_{x, m}\left(T_{x}\right)$ of recurrent times $T_{x}$ for three experimental catalogs (A2, B1 and B3) varying the moment threshold $m$. A collapse of the data is observed for intermediate $T_{x}$ values. The straight line has a slope of 0.95. Contrary to Davidsen et al. (Davidsen et al. (2006)) $P_{m, x}\left(T_{x}\right)$ is not independent of $m$ : the cut-off indicating a lower limit of the recurrent times varies with this threshold value. A fanning of the curves can be observed for large $T$ with the varying moment thresholds. For increasing $m$ value, the range of the power-law regime decreases. Bak et al. (Bak et al. (2002)) also showed a deviation from the power-law regime for large waiting times, but in their case, the range of the linear regime increases with the limiting value $m$. The shift of the distributions from the power law of slope 0.95 for the experimental catalogs is probably due to the incompleteness of these catalogs. The lack of really big events is closely related to the front propagation velocity or to the local variations of the toughness on the interface.

\section{Figure 11}




\section{Discussion}

In the previous section, we compared the spatial and temporal analyses made on the experimental quake catalogs and that applied to seismicity catalogs. The distributions of size of quakes and epicenter jumps obey power laws like the distributions of magnitude and spatial distances between epicenters of earthquakes. The record breaking analysis of the experimental catalogs and that of the SHLK catalog also show strong analogies: the distributions of distances and waiting times between a quake and its recurrent events both follow a power law decay. Furthermore the values of the scaling law exponents for the experimental catalogs and real seismicity data are really close. A good data collapse can be observed between the two experiments performed on two samples with different original sand-blasting (200 $\mu m$ and 500

$\mu m$ bead size). No sign of a possible influence of the preparation difference can be found in the graphs. However more experiments on samples sand-blasted with various sized beads are needed to draw a significant conclusion on the influence of the size of disorder introduced over the crack propagation. All these scaling properties show that the dynamics of crack propagation at large scales is controlled by microstructures within the material. The good agreement between experimental and real seismicity catalogs tends to suggest that the global dynamics of rupture propagation depends on the interactions between events. These interactions are controlled by the long range elastic coupling between heterogeneous microstructures (Ramanathan and D. (1998); Schmittbuhl et al. (2003a,b)).

One main point of discussion for the comparison between the experimental catalogs and real seismicity data is the fracturing mode. Indeed an earthquake is modelled by a mode II or III shear rupture whereas the fracture experiment described in this paper is in mode I (opening). Attempts were already made to find analogies between mode I and mode II/III fracturation. Gao and Rice (1989) showed that mathematically the 2-D problem of an antiplane rupture along a frictional fault is governed by the same equations as the mode I perturbed crack front problem. The numerical model of crack front propagation of Schmittbuhl et al. (2003a) based on that of Gao and Rice (1989) was compared to former experiments based on the same mechanical setup described in this paper. Here the experimental data are directly compared to real seismicity data assuming an analogy between the moment for mode I and for mode II/III. The moment is directly proportional to the area since the opening is assumed to be constant (the elastic deformation gradient at the crack tip is supposed to be on a very small region considering the 
size of an event). This is not the case for the seismic moment where the fault offset $D$ varies.

In conclusion, the quake catalogs derived from the optical monitoring of a crack propagation experiment have strongly similar scaling properties with real seismicity catalogs. This result yields to a possible explanation of the rupture propagation mechanism: the rupture front is trapped by local asperities and its depinning involves local instabilities. Heterogeneities in the medium play thus a crucial part in the fracture propagation. The advantage of the experiment presented in this paper is the possibility to follow optically and continuously over a large range of time scales, the propagation of the rupture thanks to the use of a transparent material, which can not be done for earthquake faults. Analyses of the experimental data offer good hints to address the question of earthquake interactions in complex media.

Acknowledgements The authors would like to thank P. Srutarshi, G. Daniel and M. Bouchon for fruitful discussions about this project. This work was supported by the programs ANR CATT MODALSIS and PICS "France/Norway".

\section{References}

Aochi, H. and Fukuyama, E. (2002). Three-dimensional nonplanar simulation of the 1992 landers earthquake. Journal of Geophysical Research, 107(B2):2035.

Bak, P., Christensen, K., Danon, L., and Scanlon, T. (2002). Unified scaling law for earthquakes. Physical Review Letters, 88(17):178501.

Bouchaud, E. (1997). Scaling properties of cracks. Journal of Physics: Condensed Matter, $9(21)$.

Bouchaud, E., Lapasset, G., Planès, J., and Naveos, S. (1993). Statistics of branched fracture surfaces. Physical Review B, 48(5):2917-2928.

Bouchon, M., Toksöz, N., Karabulut, H., Bouin, M.-P., Dietrich, M., Aktar, M., and Edie, M. (2000). Seismic imaging of the 1999 izmit (turkey) rupture inferred from the near-fault recordings. Geophysical Research Letters, 27(18):3013-1016.

Clinton, J., Hauksson, E., and Solanki, K. (2006). An evaluation of the scsn moment tensor solutions: robustness of the mw magnitude scale, style of faulting and automation of the method. Bulletin of the Seismological Society of America, 96(5):1689-1705. 
Corral, A. (2004). Long-term clustering, scaling and universality in the temporal occurence of earthquakes. Physical Review Letters, 92(10):108501.

Daguier, P., Bouchaud, E., and Lapasset, G. (1995). Roughness of a crack front pinned by microstructural obstacles. Europhysical Letters, 30(7):367-372.

Davidsen, J., Grassberger, P., and Paczuski, M. (2006). Earthquake recurrence as a record breaking process. Geophysical Research Letters, 33:L11304.

Davidsen, J. and Paczuski, M. (2005). Analysis of the spatial distribution between successive earthquakes. Physical Review Letters, 94:048501.

Delaplace, A., Schmittbuhl, J., and Måløy, K. (1999). High resolution description of a crack front in a heterogeneous plexiglas block. Physical Review Letters, 60(2):1337-1343.

Fukuyama, E., Mikumo, T., and Olsen, K. (2003). Estimation of the critical slip-weakening distance: theoritical background. Bulletin of the Seismological Society of America, 93(4):18351840.

Gao, H. and Rice, J. (1989). A first-order perturbation analysis of crack trapping by arrays of obstacles. ASME Journal of Applied Mechanics, 56:828-836.

Gutenberg, B. and Richter, C. (1944). Frequency of earthquakes in california. Bulletin of the Seismological Society of America, 34:185-188.

Ide, S. and Takeo, M. (1997). Determination of constitutive relations of fault slip based on seismic wave analysis. Journal of Geophysical Research, 102(B12):27379-37391.

Kagan, Y. (1999). Universality of the seismic moment-frequency relation. Pure and Applied Geophysics, 155(2):641-655.

Kagan, Y. (2002). Aftershock zone scaling. Bulletin of the Seismological Society of America, 92:537-573.

Kanamori, H. (1977). The energy release in great earthquakes. Journal of Geophysical Research, 82(B20):2981-2988.

Kanamori, H. and Anderson, D. (1975). Theoritical basis of some empirical relations in seismology. Bulletin of the Seismological Society of America, 65(5):1073-1095. 
Kikuchi, M. and Kanamori, H. (1991). Inversion of complex body waves. Bulletin of the Seismological Society of America, 81(6):2335-2350.

Mai, P. and Beroza, G. (2000). Source scaling properties from finite-fault-rupture models. Bulletin of the Seismological Society of America, 90(3):604-615.

Mai, P. and Beroza, G. (2002). A spatial random field model to characterize complexity in earthquake slip. Journal of Geophysical Research, 107(B11):2308.

Måløy, K., Santucci, S., Schmittbuhl, J., and Toussaint, R. (2006). Local waiting time fluctuations along a randomly pinned crack front. Physical Review Letters, 96(4).

Måløy, K., Toussaint, R., and Schmittbuhl, J. (2005). Dynamics and structure of interfacial crack fronts. Proceedings of the 11th International Congress on Fracture, page 6.

Mandelbrot, B., Passoja, D., and Paullay, A. (1984). Fractal character of fracture surfaces of metal. Nature, 308:721-722.

Marsan, D., Bean, C., Steacy, S., and McCloskey, J. (2000). Observation of diffusion processes in earthquake populations and implications for the predictability of seismicity systems. Journal of Geophysical Research, 105(B12):28081-28094.

Ramanathan, S. and D., F. (1998). Onset of propagationi of planar cracks in heterogeneous media. Phys. Rev.B, 58(10):6026-6046.

Reid, H. (1910). The california earthquake of april 18, 1906. In Report of the state earthquake investigation committee, volume II. Carnegie Institution of Washington.

Rubin, A. and Ampuero, J.-P. (2007). Aftershock asymmetry on a bimaterial interface. Journal of Geophysical Research, 112:B05307.

Santucci, S., Måløy, K., Toussaint, R., and Schmittbuhl, J. (2006). Self-affine scaling during interfacial crack front propagation. In Proceedings of Dynamics of Complex Interconnected Systems: Networks and Bioprocesses NATO ASI Geilo. Springer.

Santucci, S., Mathiesen, J., Måløy, K., Hansen, A., Schmittbuhl, J., Vanel, L., Delaplace, A., Haavig Bakke, J., and Ray, P. (2007). Statistics of fracture surfaces. Physical Review E, 75(1):016104. 
Schmittbuhl, J., Delaplace, A., and Måløy, K. (2001). Propagation of an interfacial crack front in an heterogeneous medium: experimental observations. In Proceedings of the NATO Advanced Study Institute on Physical Aspects of Fracture, pages 353-369. Kluwer Academic Publishers.

Schmittbuhl, J., Delaplace, A., Måløy, K., Perfettini, H., and Vilotte, J. (2003a). Slow crack propagation and slip correlations. Pure and Applied Geophysics, 160.

Schmittbuhl, J., Gentier, S., and Roux, S. (1993). Field measurements of the roughness of fault surfaces. Geophysical Research Letters, 20(8):639-641.

Schmittbuhl, J., Hansen, A., and Batrouni, G. (2003b). Roughness of interfacial crack fronts: stress-weighted percolation in the damage zone. Physical Review Letters, 90(4).

Schmittbuhl, J. and Måløy, K. (1997). Direct observation of a self-affine crack propagation. Physical Review Letters, 78(20):3888-3891.

Schmittbuhl, J., Schmitt, F., and Scholz, C. (1995). Scaling invariance of crack surfaces. Journal of Geophysical Research, 100(B4):5953-5973.

Scholz, C. (2002). The mechanics of earthquakes and faulting. Cambridge Univ. Press, second edition.

Shearer, P., Hauksson, E., and Lin, G. (2005). Southern california hypocenter relocation with waveform cross-correlation, part 2: results using source-specific station terms and cluster analysis. Bulletin of the Seismological Society of America, 95(3):904-915.

Tinti, E., Spudich, P., and Cocco, M. (2005). Earthquake fracture energy inferred from kinematic rupture models on extended faults. Journal of Geophysical Research, 110:B12303.

Wald, D., Helmberger, D., and Heaton, T. (1991). Rupture model of the 1989 loma prieta earthquake from the inversion of strong-motion and broadband teleseismic data. Bulletin of the Seismological Society of America, 81(5):1540-1572.

Wells, D. and Coppersmith, K. (1994). New empirical relationships among magnitude, rupture length, rupture width, rupture area and surface displacement. Bulletin of the Seismological Society of America, 84(4):974-1002. 
Ziv, A., Rubin, A., and Kilb, D. (2003). Spatiotemporal analyses of earthquake productivity and size distribution: observations and simulations. Bulletin of the Seismological Society of America, 93(5):2069-2081. 


\begin{tabular}{|c|c|c|c|c|c|c|}
\hline Experiment & $\varnothing(\mu m)$ & $\mathrm{Nb}$ of images & $\langle v\rangle\left(\mu m s^{-1}\right)$ & C levels & $\mathrm{Nb}$ of clusters & Catalog \\
\hline \multirow{3}{*}{$\mathrm{A}$} & \multirow{3}{*}{200} & \multirow{3}{*}{5908} & \multirow{3}{*}{214} & 5 & 1990 & A1 \\
\hline & & & & 10 & 2454 & $\mathrm{~A} 2$ \\
\hline & & & & 20 & 2583 & A3 \\
\hline \multirow{3}{*}{ B } & \multirow{3}{*}{500} & \multirow{3}{*}{8001} & \multirow{3}{*}{295} & 3 & 4756 & B1 \\
\hline & & & & 7 & 6423 & B2 \\
\hline & & & & 10 & 6321 & B3 \\
\hline
\end{tabular}

Table 1: Table of parameters for the two experiments described in the article. Nb stands for number. $\varnothing$ is the diameter of the beads used for the sand-blasting of the sample. 


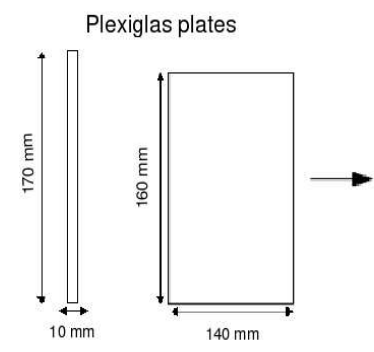

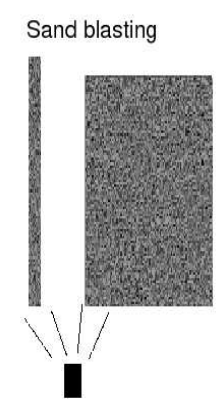

(a)

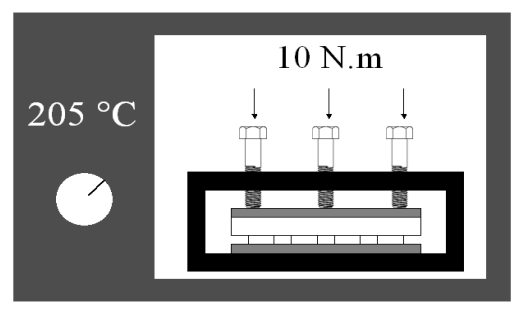

(b)

Figure 1: (a) Sample preparation procedure. Plexiglas plates are sand-blasted and sintered together to form a new single block. Sandblasting creates roughness fluctuations on the plate surface that control the local toughness during the annealing process. It also induces a loss of transparency by unpolishing of the surfaces. Transparency is however recovered thanks to the sintering process. (b) Scheme of the loading system: in white, the PMMA sample; in gray, the two aluminium plates; in black, the solid frame with the screws to apply a torque $N=10$ N.m. The whole block is put in an oven at $205^{\circ} \mathrm{C}$ during 30 minutes. 

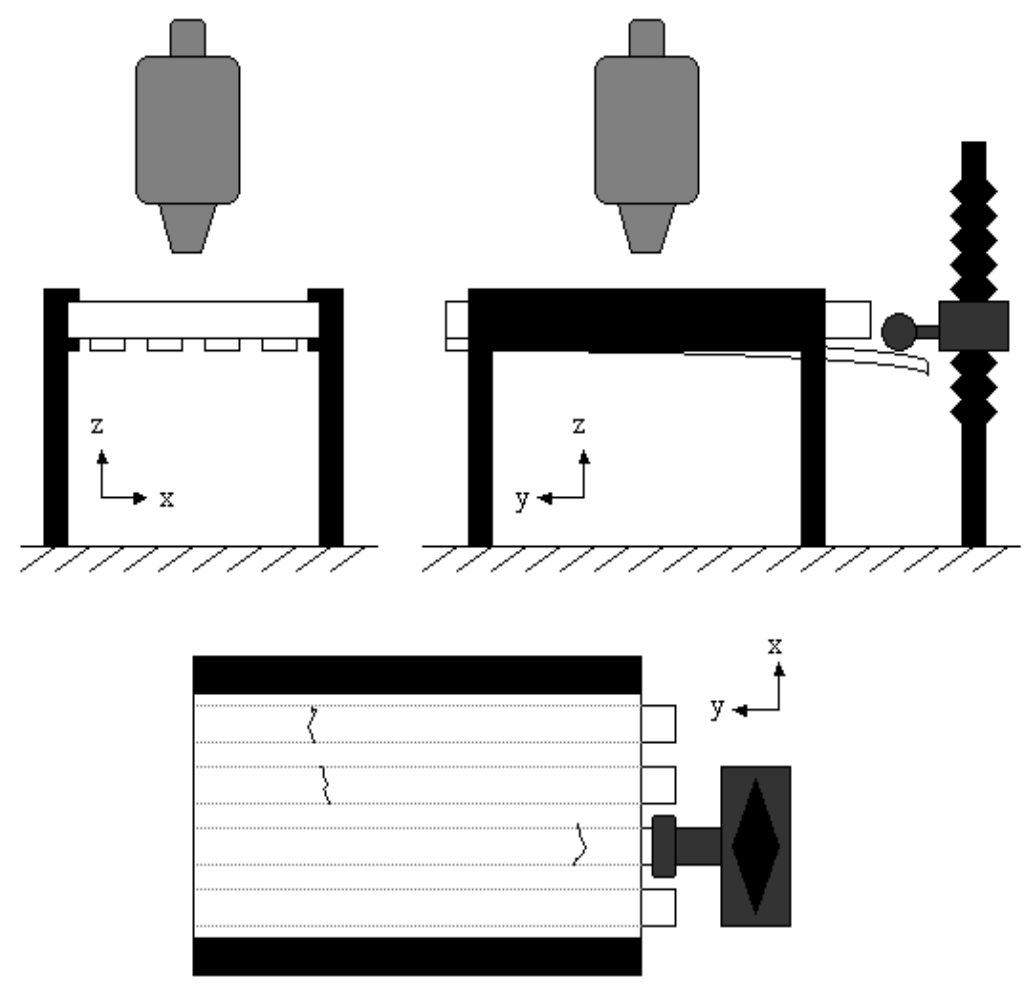

Figure 2: Sketch of the experimental setup (top left: Front view; top right: Side view; bottom: View from above). The thick plate of the sample (in white) is clamped into a rigid aluminium frame (in black). A cylindrical rod imposes a displacement of the thin Plexiglas band. The crack propagates in the $y$ direction and is observed from above using a high speed camera mounted on a microscope (in light gray). 


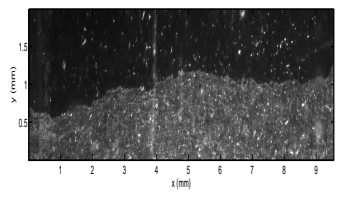

(a)

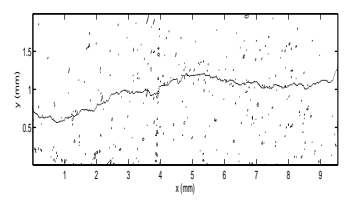

(e)

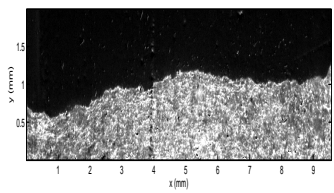

(b)

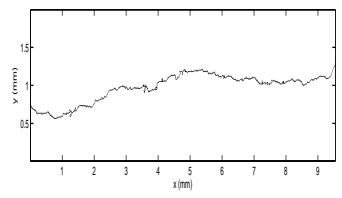

(f)

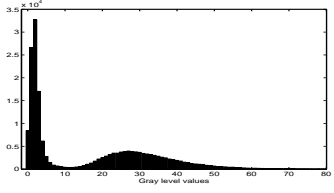

(c)

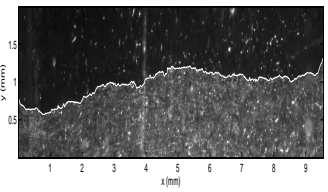

(g)

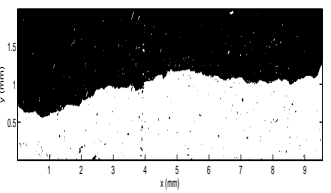

(d)

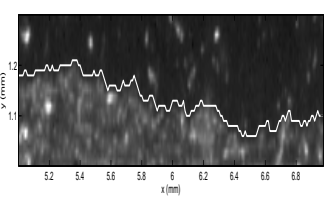

(h)

Figure 3: (a) Example of raw image taken with the high speed camera. (b) Example of the resulting picture of a subtraction between two raw images. (c) Gray level histogram of the preceding picture. (d) Image after thresholding. (e) Gradient of the clipped image. (f) Extraction of the percolating cluster from the gradient picture. (g) Extracted front (white line) superimposed on its corresponding raw image. (h) Zoom on the extracted front. 


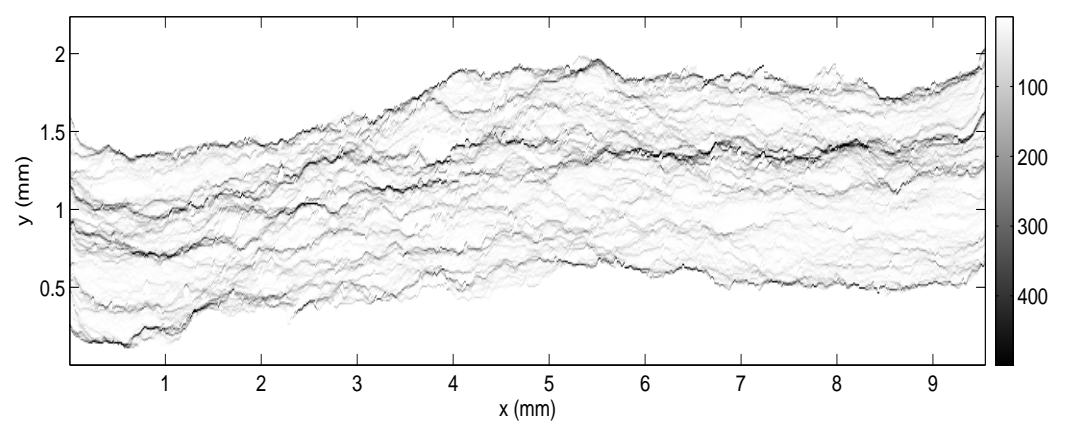

(a)

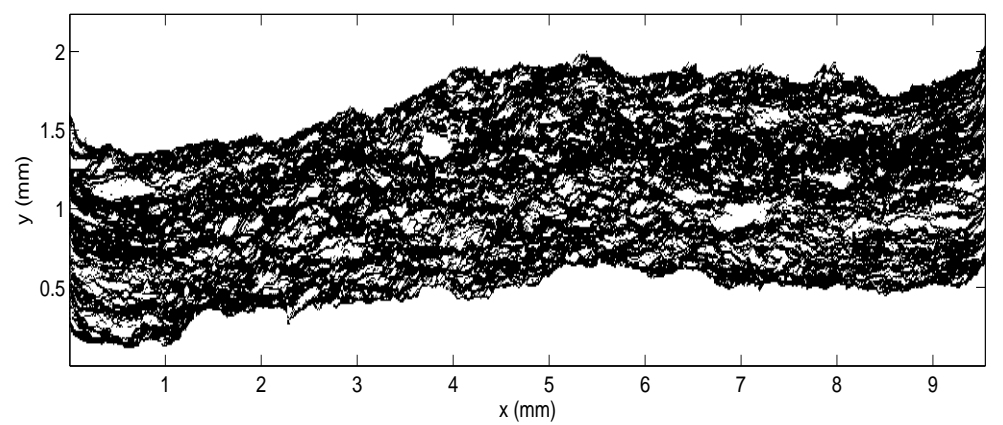

(b)

Figure 4: (a) Gray scale map of the waiting time matrix for one experiment deduced from 5908 fronts recorded at a rate of 1000 fps. The darker spots show the longer waiting times. (b) Spatial distribution of clusters (in white) for velocities 10 times larger than the average front speed (here $\left.\langle v\rangle=214 \mu \mathrm{ms}^{-1}\right)$. 


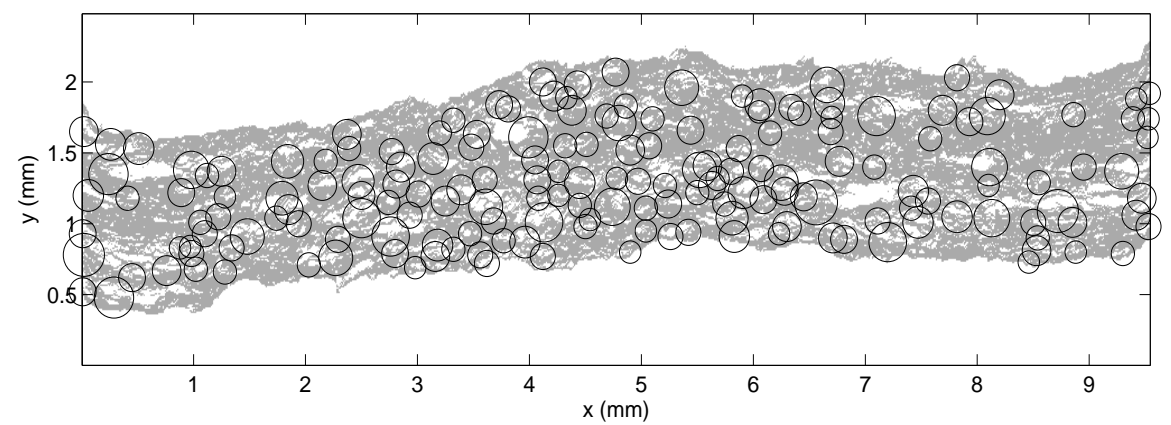

Figure 5: Epicenter localisation superimposed on the thresholded velocity matrix for experiment A2. The diameters of the circles are proportional to the logarithm of the area of the clusters. Only events with $\log _{10}(M)>1.5$ are shown for a better visibility. 


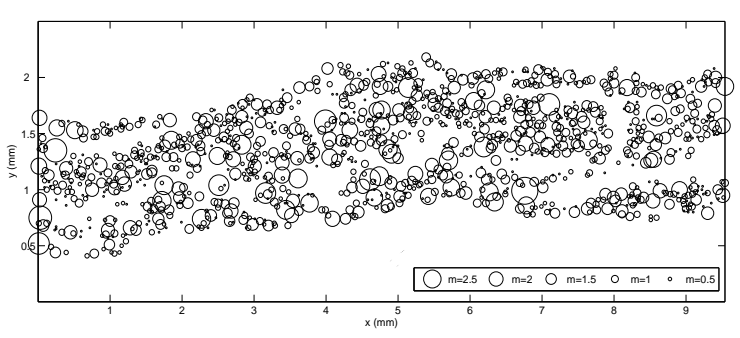

(a)

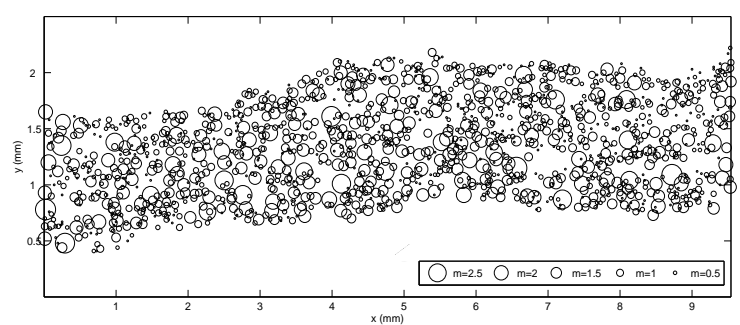

(b)

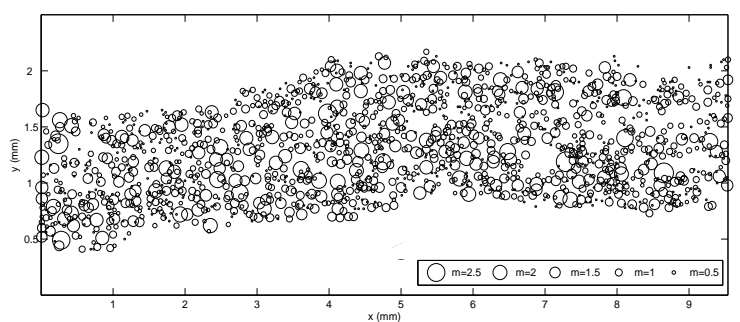

(c)

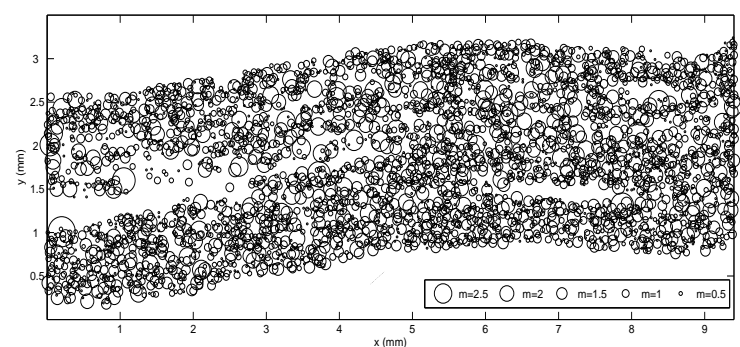

(d)

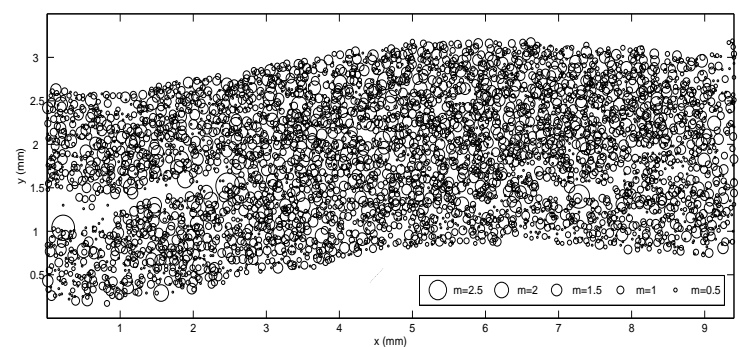

(e)

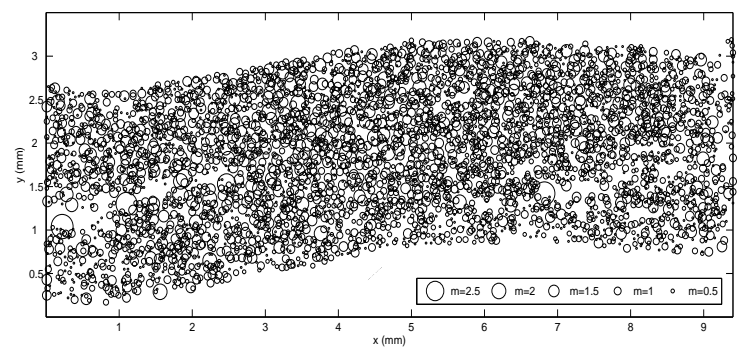

(f)

Figure 6: Epicenters localisation of events for the six experimental catalogs. The diameters of the circles are proportional to the logarithm $m$ of the area of the clusters. (a) Catalog A1. (b) Catalog A2. (c) Catalog A3. (d) Catalog B1. (e) Catalog B2. (f) Catalog B3. 


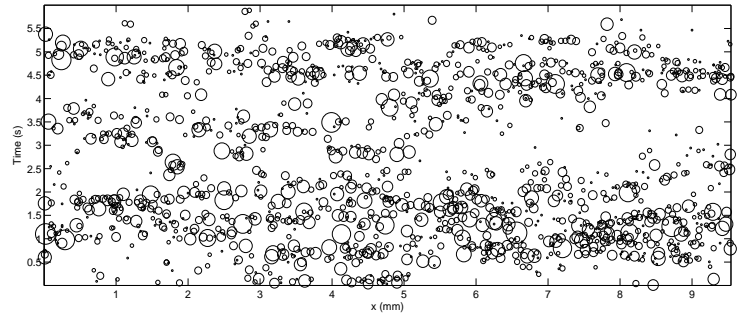

(a)

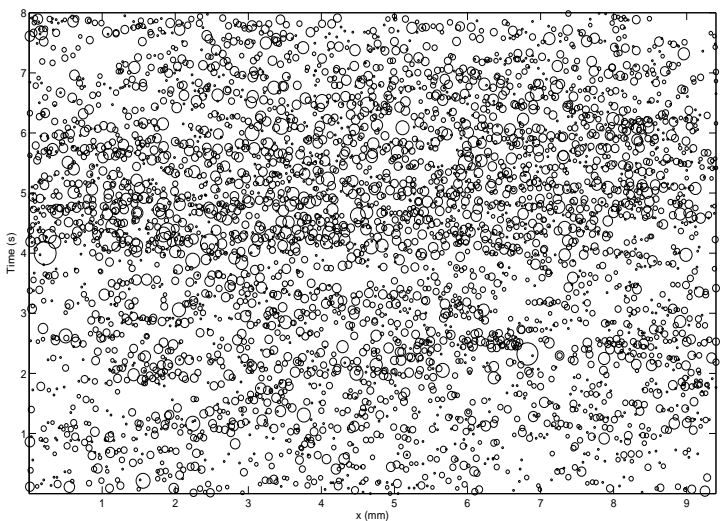

(b)

Figure 7: Spatio-temporal distribution of events for two experimental catalogs: (a) A2 and (b)

B3. The diameter of the circles representing the events is proportional to the parameter $m$. 


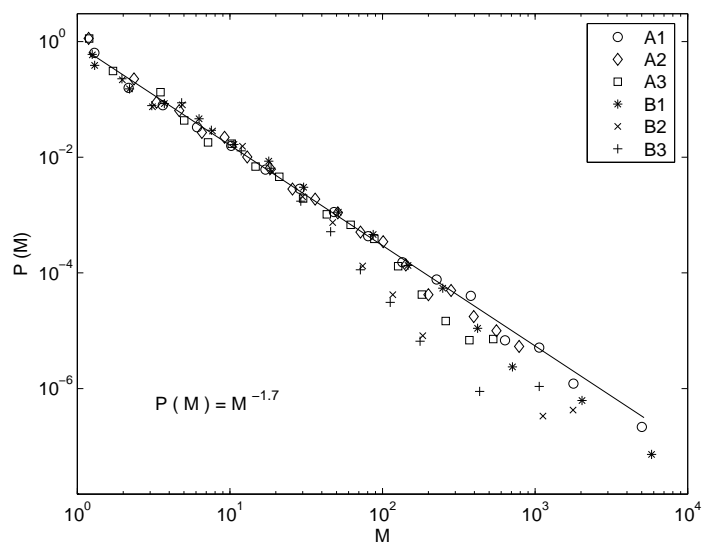

(a)

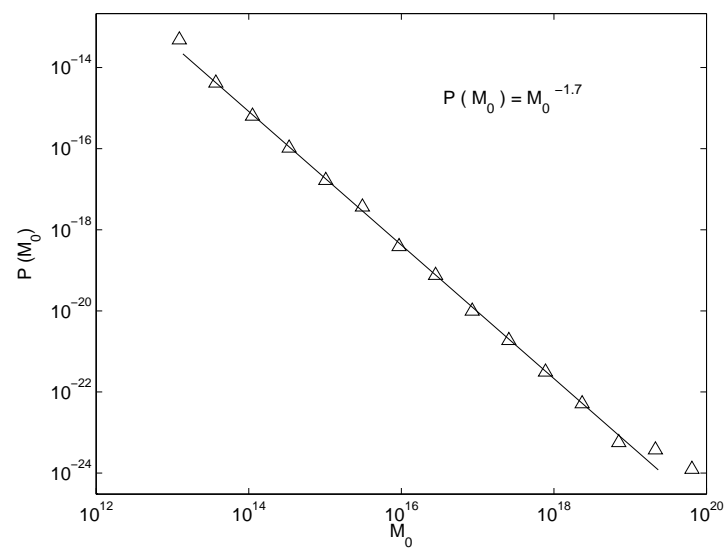

(b)

Figure 8: (a) Burst size $M$ distributions $P_{M}$ for the six experimental catalogs. A fit on all the data for $0 \leq m \leq 2$ (black line) gives a slope of 1.7. A cut-off appears around $m=2$ for large $\mathrm{C}$ values because of the lack of statistics for the largest clusters. (b) Seismic moment distribution $P_{M_{0}}$ for a subset of the Californian seismicity catalog. The dot-dashed curve shows a fit of the data with a slope equal to 1.7 . 


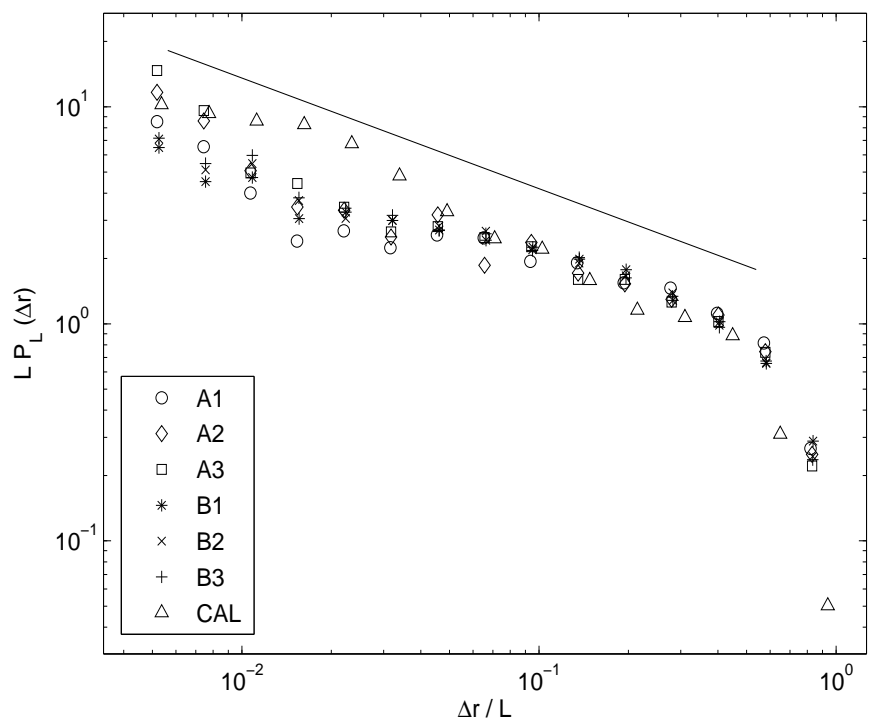

Figure 9: Distribution of jumps $P_{L}(\Delta r)$ for the six experimental catalogs and a subset of the Southern California seismicity catalog rescaled by the longest size of the considered area $L$. Distances $\Delta r<2 k m$ for the Californian catalog and $\Delta r<40 \mu m$ for the experimental catalogs have been discarded because of uncertainties in the locations of hypocenters. The solid black line has a slope $\gamma=0.6$ and is shown as a guide to the eye. 


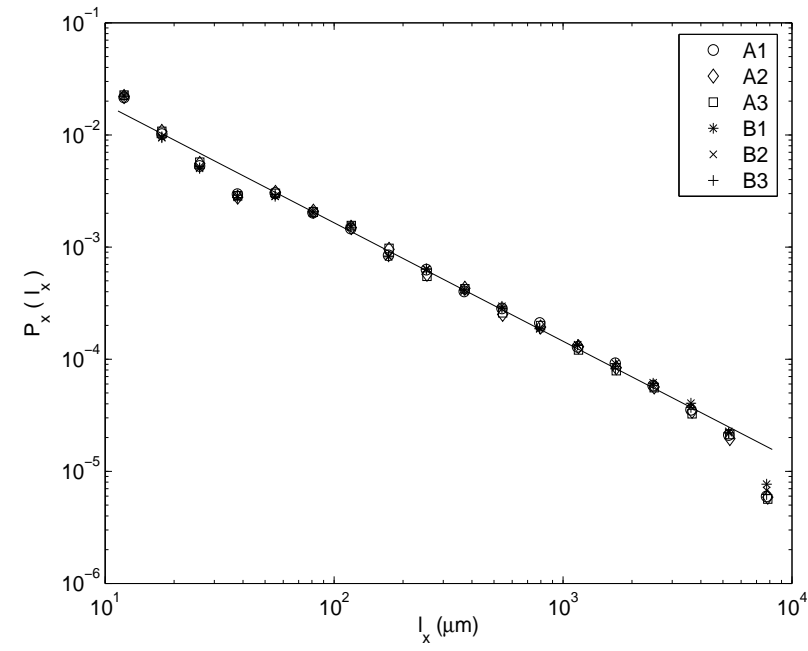

(a)

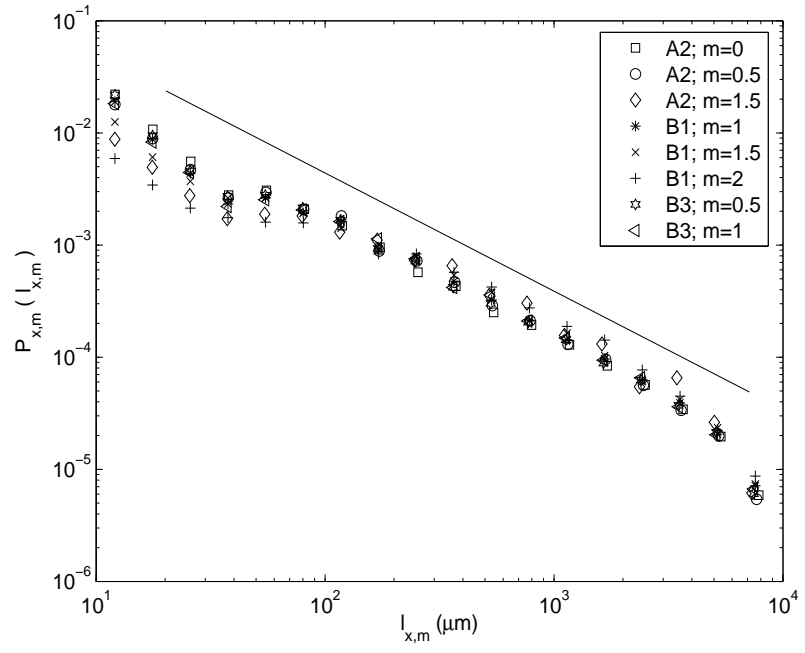

(b)

Figure 10: (a) Distribution $P_{x}\left(l_{x}\right)$ of distances $l_{x}$ of recurrent events considering only the positions of epicenters on the x-axis for the six experimental catalogs. The straight line has a slope of 1.1. (b) Distribution $P_{x, m}\left(l_{x}\right)$ of distances $l_{x}$ for three catalogs (A2, B1 and B3) varying the threshold moment $m$. Only events with moments greater or equal to $m$ are considered. Data for $m=0$ cover the whole catalog. The black line corresponds to a power law with an exponent equal to 1.1. 


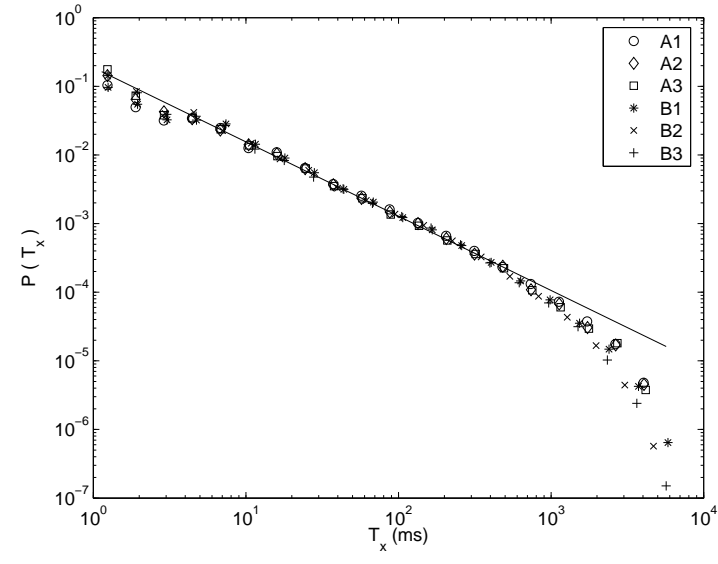

(a)

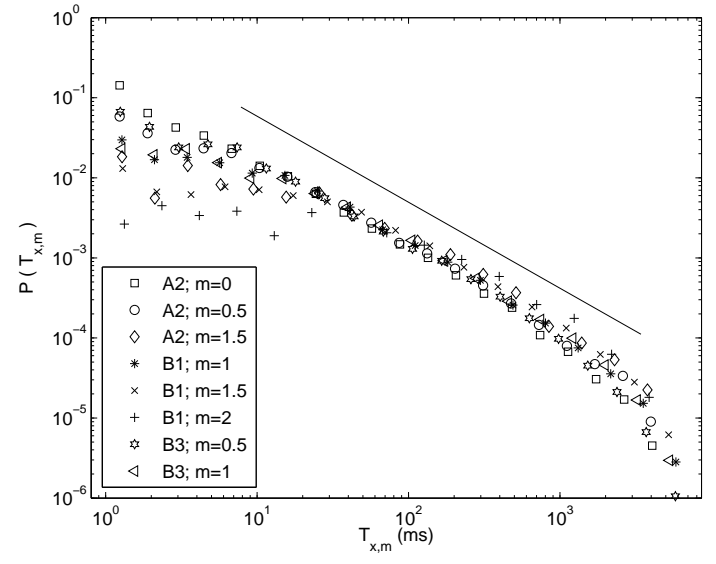

(b)

Figure 11: (a) Distribution $P_{x}\left(T_{x}\right)$ of recurrent time intervals $T_{x}$ for the six experimental catalogs when analysed considering only the x-coordinate position of the events. The straight line has a slope $\approx 0.95$. (b) Distribution $P_{x, m}\left(T_{x}\right)$ of waiting times $T_{x}$ for three catalogs $(\mathrm{A} 2$, B1 and B3) varying the threshold moment $m$. Only events with moments larger or equal to $m$ are taken into account. For $m=0$ the whole catalog is involved. The black line corresponds to the power law with a slope of 0.95 . 